\title{
Coastal numerical modelling of tides: Sensitivity to domain size and remotely generated internal tide
}

\author{
Aurelien L. Ponte ${ }^{a, *}$, Bruce D. Cornuelle \\ a Laboratoire de Physique des Océans, IFREMER, 29280 Plouzané, France \\ ${ }^{b}$ Scripps Institution of Oceanography, UCSD, United States \\ *: Corresponding author: Aurélien L. Ponte, Tel.: +33298224040 ; fax: +33298224496 ; \\ email address : aurelien.ponte@ifremer.fr
}

\begin{abstract}
:
The propagation of remotely generated superinertial internal tides constitutes a difficulty for the modelling of regional ocean tidal variability which we illustrate in several ways.

First, the M2 tidal solution inside a control region located along the Southern California Bight coastline is monitored while the extent of the numerical domain is increased (up to $512 \times 512 \mathrm{~km}$ ). While the amplitude and phase of sea level averaged over the region is quasi-insensitive to domain size, a steady increase of kinetic energy, predominantly baroclinic, is observed with increasing domain size. The increasing flux of energy into the control region suggests that this trend is explained by the growing contribution from remote generation sites of internal tide which can propagate up to the control region.
\end{abstract}

Increasing viscosities confirms this interpretation by lowering baroclinic energy levels and limiting their rate of increase with domain size. Doubling the grid spacing allows consideration of numerical domains 2 times larger. While the coarse grid has lower energy levels than the finer grid, the rate of energy increase with domain size appears to be slowing for the largest domain of the coarse grid simulations.

Forcing the smallest domain with depth-varying tidal boundary conditions from the simulation in the largest domain produces energy levels inside the control region comparable to those in the control region for the largest domain, thereby confirming the feasibility of a nested approach.

In contrast, simulations forced with a subinertial tidal constituent (K1) show that when the propagation of internal tide is limited, the control region kinetic energy is mostly barotropic and the magnitudes of variations of the kinetic energy with domain size are reduced.

\section{Highlights}

We assess the sensitivity of the tidal response in a coastal region to domain size. The M2superinertial tidal kinetic energy grows with domain size. This is due to the growing contribution from remotely generated internal tides. The kinetic energy growth for a subinertial tidal consituent (K1) is slower. 
Keywords: Barotropic tide ; Baroclinic tide ; Internal tide ; Coastal dynamics ; Domain size sensitivity ; Energy budgets

\section{Introduction}

The oceanic input of tidal energy by astronomical forcing occurs at large spatial scales and the bulk of the response is a barotropic motion which sweeps over the ocean with phase speed exceeding $100 \mathrm{~m} \mathrm{~s}^{-1}$. In the deep ocean the associated sea level fluctuations and depth-uniform currents are of the order of $1 \mathrm{~m}$ and $1 \mathrm{~cm} \mathrm{~s}^{-1}$, respectively. Tide gauges and satellite altimetry have allowed a detailed mapping of the barotropic response and a better understanding of its dissipation, one third of which is due to the production of baroclinic tidal motion (Egbert and Ray, 2003).

Baroclinic tidal fluctuations are produced when barotropic currents flow across a bathymetric slope and isopycnals are disturbed (Garrett and Kunze, 2007). Guided by maps of barotropic tidal dissipation from satellite altimetry (Egbert and Ray, 2001), observational campaigns near internal tide generation hotspots and numerical simulations have improved our understanding of the generation process over the last decade (Klymak et al., 2006, Legg and Huijts, 2006 and Carter et al., 2008). A small fraction of the energy dissipates locally. Most of the energy radiates away as a low mode internal wave (Laurent and Garrett, 2002). For the semidiurnal tide, wavelengths are about $150 \mathrm{~km}$ and group speeds are below $<3 \mathrm{~m} \mathrm{~s}^{-1}$ (Alford and Zhao, 2007). The low mode waves can propagate over $O(1000 \mathrm{~km})$ distances (Dushaw et al., 1995, Ray and Mitchum, 1997, Alford et al., 2007 and Zhao and Alford, 2009) and the mechanisms for their ultimate decay are a topic of ongoing debate: bathymetric scattering into higher modes (Bühler and Holmes-Cerfon, 2011), dissipation against coastal boundaries where areas with critical bathymetric slope are abundant ( Nash et al., 2004, Martini et al., 2011 and Kelly et al., 2012), and nonlinear interaction with the internal wave spectrum (Hazewinkel and Winters, 2011).

Internal tide fluctuations are energetic in the coastal ocean. They are important to marine biology (Lucas et al., 2011), sediment transport (Heather- 
shaw, 1985), lateral heat flux and mass transport (Inall et al., 2001; Shroyer et al., 2010), mixing (Sharples et al., 2007), and acoustic propagation (Duda and Preisig, 1999). The preceding list highlights the need for proper description and prediction of the tidal variability in the coastal domain.

The long range propagation of tidal fluctuations represents an underestimated challenge for the coastal modeling of tides. A typical study of the three-dimensional tide along the coast uses tidal sea level and current from an assimilation product based on barotropic dynamics (Kurapov et al., 2003; Rosenfeld et al., 2009; Pairaud et al., 2010; Carter, 2010). The effect of remotely generated internal tide is not taken into account, the assumption being that local generation, generally at the shelf break, dominates the variability. This assumption could be justified by the enhanced topographic roughness close to the coasts which could facilitate the reflection and/or scattering and dissipation of remotely generated baroclinic tides before reaching the area of interest. There is evidence that this is not true in general (Martini et al., 2011; Kelly et al., 2012) and it is therefore necessary to verify this assumption, potentially on a case by case basis. One would ideally extend the numerical domain in order to include all possible remote generation sites, but computational resources are ultimately limiting. The present study cannot conclude, for example, on the importance of internal wave sources located more than $1000 \mathrm{~km}$ away from our region of interest. Few numerical experiments have investigated the sensitivity of tidal simulations to domain size. Hall and Carter (2011) used simulations on two domains of different sizes (up to $180 \times 180 \mathrm{~km}$ ) and showed that energy fluxes into the Monterey Canyon are greatest with the larger domain. The present study finds similar results for a different geographical location, extending the results of Hall and Carter (2011). We additionally consider larger domains and investigate how model parameters such as grid spacing and viscosities affect the contribution from remotely generated fluctuations.

We select a control region located in the Southern California Bight offshore San Diego, California and monitor the sensitivity of the local tidal solution to model domain. Most reports on tidal variability in this area have been in depths shallower than $100 \mathrm{~m}$, over the mainland continental shelf (Winant and Bratkovich, 1981; Bratkovich, 1985; Noble et al., 2009; Lucas et al., 2011). Lerczak et al. (2003) reports on observations over the shelf as well as over the shelf break down to $300 \mathrm{~m}$ depths. A common feature of the shelf variability is that currents do not tend to follow the spring neap cycle. Lerczak et al. (2003) describes the structure of semidiurnal shelf currents as 


\section{Model setup}

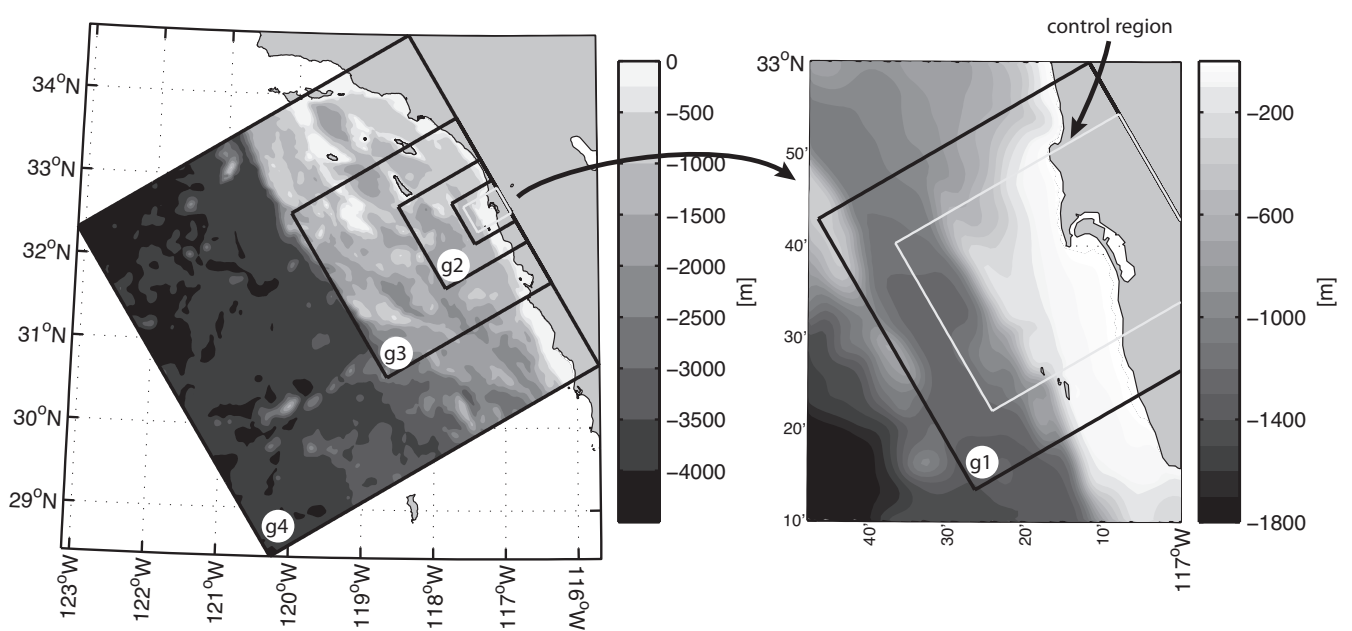

Figure 1: Numerical domains (black) and control region (white). Shading shows the water depth in meters. Right is a zoom on the control region.

The control region used by the present study is that of Hoteit et al. (2009), a 30 by $40 \mathrm{~km}$ rectangle around Point Loma in San Diego, California (white 
rectangle in Fig. 1). The numerical calculations have used the $M I T g \mathrm{~cm}$ (Marshall et al., 1997). A set of overlapping numerical domains was chosen such that the eastern edge of the control region is centered on the eastern edge of each domain. The size of the domain doubles from one to the next, from 64 by $64 \mathrm{~km}$ (g1, barely bigger than the target area) to 512 by $512 \mathrm{~km}(\mathrm{~g} 4)$. The model is run with spherical coordinates and the horizontal grid spacing is approximately $1 \mathrm{~km}$. Vertical grid spacing varies from $1 \mathrm{~m}$ close to the surface to $30 \mathrm{~m}$ at depth and is the same for all grids. Because the maximum depth increases with numerical domain size, the number of vertical levels varies from 115 (g1) to 200 (g4). Model bathymetry is obtained from the NGDC's 3 arc-second U.S. Coastal Relief Model when available. Elsewhere ETOPO1 (Amante and Eakins, 2009) is linearly interpolated to the model grid. The overlapped grids are aligned so that grid points are collocated horizontally and vertically, and bathymetry is identical in the overlapping portions of the domains. Initial stratification is horizontally uniform, taken from a winter average of CALCOFI station number 28 (http://www.calcofi.org), closest to the target domain (Fig. 1). Below $500 \mathrm{~m}$ the temperature and salinity from the 2005 World Ocean Atlas (Locarnini et al., 2006; Antonov et al., 2006) at a nearby deepwater location is used to complete the profile.

Tidal forcing is applied at the boundaries, where the sea level is prescribed. Along-boundary and cross-boundary currents are relaxed on the boundaries to tidally fluctuating values with a $1000 \mathrm{~s}$ time scale. This approach differs from the default MITgcm open boundary conditions where the flows normal and tangential to boundaries are prescribed and the sea level adjusts to the flow through boundaries (no boundary values need to be provided for sea level). With this default treatment, the M2 sea level averaged inside the control domain varies with domain size by as much as $8 \mathrm{~cm}$ in amplitude and $17^{\circ}$ in phase. This is to compare with $2.5 \mathrm{~mm}$ and $0.3^{\circ}$ when sea level is prescribed along boundaries (see section 3.2). Note that the choice of default treatment of boundary conditions or prescription of sea level does not affect energy levels by more than $15 \%$. None of the results relative to energy levels presented in this manuscript are qualitatively modified if the default treatment of boundary conditions had been used.

For the largest domain, g4, the model is forced with sea level and barotropic current from the ENPAC tidal database (Spargo et al., 2003). Smaller domains (g1 to g3) are forced by tidal-frequency sea level and currents from a simulation with fixed tracers (g4_noTS see below) on the largest domain. This is done to maintain, at least for the fixed tracer simulations, consis- 
tent barotropic dynamics between experiments on different domains. When tracers are freely evolving however, the barotropic dynamics adjusts to some extent from one domain to the next and accommodates for the loss of energy to the internal tide (see section 5.2).

Temperature and salinity are relaxed to initial profiles within nudging layers along open boundaries. The width of these nudging layers is $5 \mathrm{~km}$ for $\mathrm{g} 1$ and $10 \mathrm{~km}$ for g2 to g3 and the relaxation time scale is $1000 \mathrm{~s}$. The value of the relaxation time scale is set to be smaller than the time for a mode 1 baroclinic wave to cross the width of the nudging layers $(\sim 3000 \mathrm{~s}$ for a width of $5 \mathrm{~km}$ ). Sensitivity tests to the relaxation time scale with domain g1 indicate that a value of $1000 \mathrm{~s}$ is optimal to minimize baroclinic wave reflections.

The simulations were spun up from rest with the forcing ramped up to full strength over a period of 5 days to reduce transients. The time stepping of sea level is implicit with a $90 \mathrm{~s}$ time step. The models are run over a time period of 15 days. Harmonic vertical and horizontal viscosities are constant and with values of $210^{-3}$ and $10 \mathrm{~m}^{2} \mathrm{~s}^{-1}$, respectively. This choice aims at damping grid scale noise and explicitly diagnosing viscous energy loss at the expense of using viscosities much higher than realistic ocean values (Legg and Klymak, 2008; Kelly et al., 2012). Other studies have used more complex turbulence parametrizations (e.g. KPP, Mellor-Yamada 2.0 or 2.5) and/or rather viscous advection schemes. Energy dissipation has to be estimated from the residual of the energy budgets when the damping of grid scale fluctuations relies on advection schemes (Kang and Fringer, 2012). In the present study, horizontal and vertical diffusivities are set to 0 and a Superbee flux limiting advection scheme for tracers is used (Roe, 1985). It introduces numerical diffusion but minimizes the erosion of the stratification that a constant diffusivity would produce even in an unforced simulation.

Diagnostic runs where temperature and salinity fields are held fixed are also carried out. These runs are labeled with the suffix _noTS. They illustrate purely barotropic responses and are therefore referred to as barotropic simulations in the text, as opposed to baroclinic simulations which have a freely evolving buoyancy. 

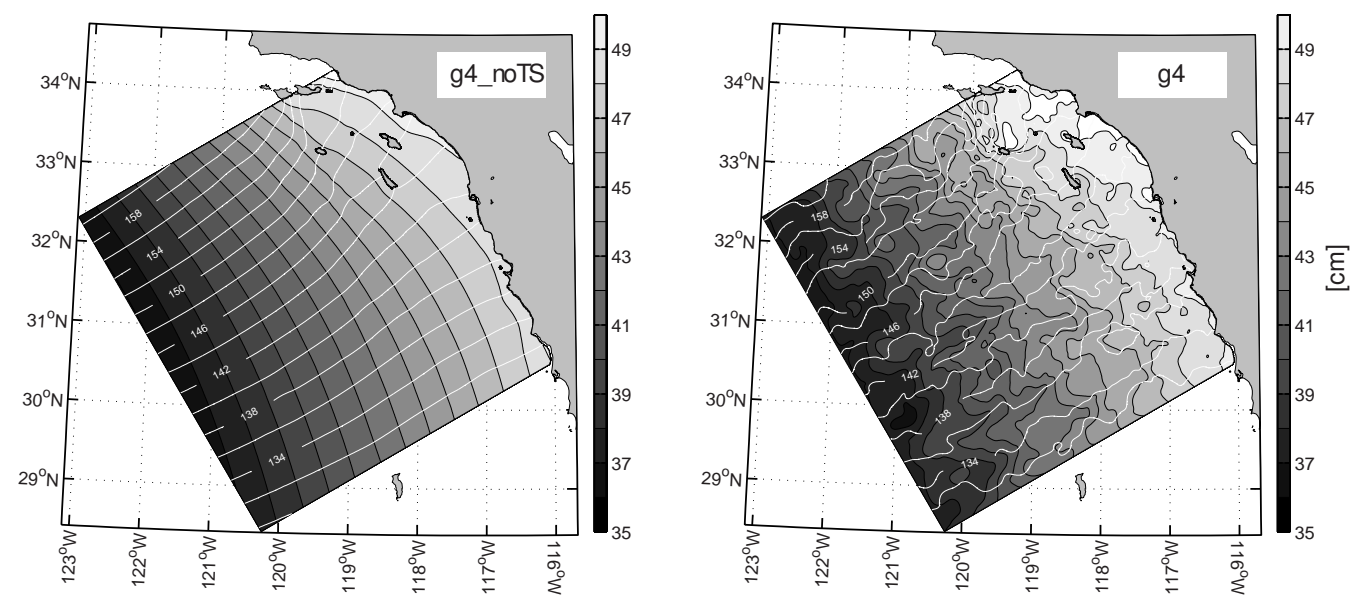

Figure 2: M2. Cotidal chart of the sea level on domain g4 for the barotropic (left) and baroclinic (right) simulations. White contours represent the phase (GMT), while shading and black contours represent the amplitude in $\mathrm{cm}$.

\section{M2 sea level response}

\subsection{Overview}

Cotidal charts of the M2 tide in the largest computational domain (g4) provide a regional overview of the tidal sea level response and illustrate the sea level signature of internal tide (Fig. 2). The tidal fits are computed over the last five days of the runs. For the barotropic simulation, isolines of amplitude and phase are smooth. The amplitude increases toward the coast while the phase progresses with the coast to the right, which is typical of Kelvin wave propagation. The northward phase increase is about $30^{\circ}$ over the length of g4, corresponding to a phase speed of approximately $137 \mathrm{~m} / \mathrm{s}$. For the baroclinic simulation, the overall pattern of amplitude and phase variation is similar. The difference with the barotropic simulation is due to a finer scale spatial variability in phase and amplitude isolines. These isolines are also displaced over distances of several tens of kilometers compared to the barotropic case. This variability is the manifestation of the internal tide on sea level (Carter, 2010).

\subsection{Sensitivity to domain size.}

When averaged over the control region, tidal amplitudes and phases of the barotropic experiments (gray lines in Fig. 3) show little sensitivity to 


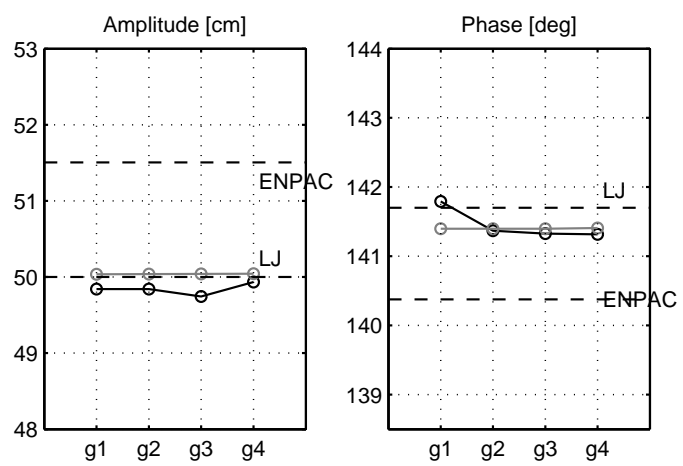

Figure 3: M2 amplitude and phase of the sea level averaged over the control region as a function of domain size. Baroclinic experiments are in black, barotropic ones in gray. Horizontal dashed lines show the M2 amplitude and phase observed at the La Jolla tide gauge (LJ) and that from the forcing barotropic model averaged inside the control region (ENPAC).

domain size. A close up look at the amplitude and phase shows variations on the order of $0.01 \mathrm{~cm}$ and $0.01^{\circ}$ respectively. These values suggest that the barotropic response is consistent between runs with different domain sizes, as further confirmed in section 4 by an inspection of kinetic energy levels. Compared to the ENPAC forcing product, the amplitude of sea level is $1.5 \mathrm{~cm}$ weaker and the phase larger by approximately $1^{\circ}(\sim 2 \mathrm{~min})$. These differences are attributed to mismatches of the bathymetry and potentially the dynamics (bottom friction for example) between ENPAC and the present numerical configuration. The magnitude of these differences could have been reduced by adjusting the forcing at the boundary.

The harmonic sea level of baroclinic experiments exhibit a greater sensitivity to domain size. This sensitivity remains weak: the tidal sea level amplitude varies around its average value by about $2 \mathrm{~mm}(0.4 \%)$. This last value is comparable to the typical tidal amplitude of scaled surface baroclinic pressure within the control region (not shown). The amplitude in baroclinic experiments is lower than that in the barotropic experiments by up to $3 \mathrm{~mm}$. The phase changes with varying domain size are up to $0.45^{\circ}$. This value is approximately consistent with the maximum phase perturbation $\delta \phi$ that can be produced by a $\delta A=2 \mathrm{~mm}$ perturbation around sea level oscillations of amplitude $A=50 \mathrm{~cm}(A \gg \delta A)$ typical of the control region. The superimposition of perturbation and background sea level fluctuations can indeed be written as: 


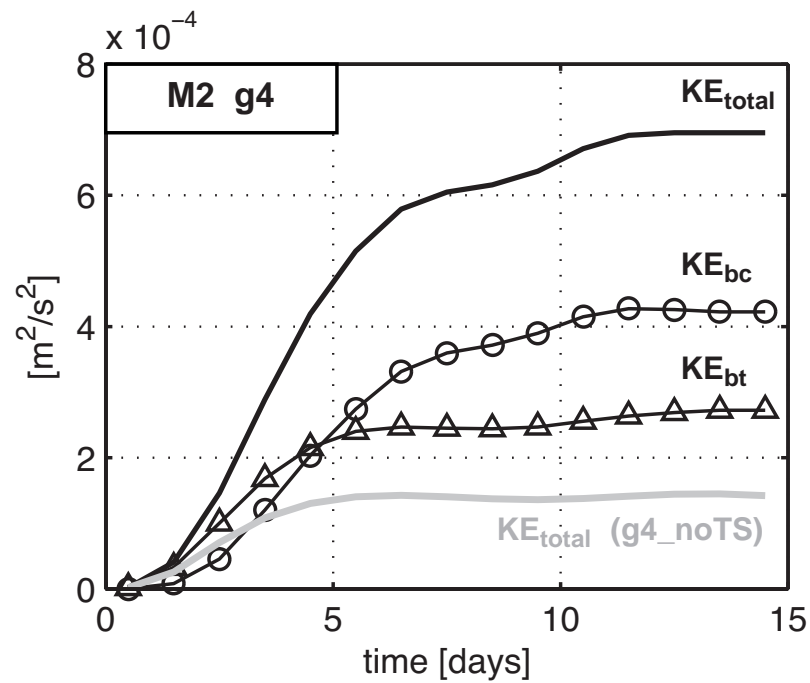

Figure 4: Volume averaged kinetic energy in the control region for runs on domain g4. A $24 \mathrm{~h}$ running mean has been applied. Full lines are the total kinetic energy, the circled and triangled lines are the baroclinic and barotropic kinetic energy, respectively. The gray line is the total kinetic energy for the barotropic experiment.

$$
A \cos (\omega t)+\delta A \sin (\omega t)=\sqrt{A^{2}+\delta A^{2}} \cos (\omega t-\delta \phi),
$$

where $\omega$ is the tidal frequency and $\delta \phi=\tan ^{-1}(\delta A / A)$. With $\delta A=2 \mathrm{~mm}$ and $A=50 \mathrm{~cm}$, the phase perturbation is approximately:

$$
\delta \phi \sim \frac{\delta A}{A} \sim 0.23^{\circ}
$$

\section{M2 kinetic energy}

\subsection{Overview}

The kinetic energy averaged over the control region shows much more sensitivity to domain size than sea level. The time evolution of total kinetic energy $\left(\mathrm{KE}_{\text {total }}\right)$ during the spin-up period for domain $\mathrm{g} 4$ shows a rapid increase over the first five days to about $70 \%$ of the final value (Fig. 4) and plateaus after about 13 days. A $24 \mathrm{~h}$ running mean was applied to remove fluctuations at the tidal frequency. 
The barotropic $\left(\mathrm{KE}_{\mathrm{bt}}\right)$ and baroclinic $\left(\mathrm{KE}_{\mathrm{bc}}\right)$ contributions to kinetic energy are also shown in Fig. 4. The barotropic-baroclinic decomposition of a variable $\phi$ follows:

where:

$$
\phi=\bar{\phi}+\phi^{\prime},
$$

$$
\bar{\phi}=\frac{1}{h} \int_{-h}^{0} \phi d z .
$$

The spin up of barotropic kinetic energy is fast with $85 \%$ of the final energy reached at day 5 while baroclinic energy has reached only $57 \%$ of its final value at that time. The baroclinic energy is the reason for the slower rise of total energy, likely due to the slower propagation and equilibration of baroclinic tide inside the domain. Consistent with this interpretation, equilibrium of the baroclinic energy level is reached faster for smaller domains (not shown). For comparison it would take 3.6/6.7/9.9 days for mode $1 / 2 / 3$ internal waves with M2 frequency to propagate over the width of g4 in an ocean with uniform depth equal to g4 mean depth $(\sim 2500 \mathrm{~m})$. Finally, Fig. 4 shows the time series of the kinetic energy of the barotropic simulation g4_noTS in gray. The spin up occurs in a fashion similar to the barotropic energy of the baroclinic experiment except that it reaches a lower equilibrium value.

\subsection{Domain size sensitivity}

The kinetic energy averaged over the control region and over day 15 is an increasing function of domain size (Fig. 5). With domain g4, the total energy in the control region is 3.1 times that with g1. This is largely explained by a factor of 4.2 increase in baroclinic kinetic energy, which is attributed to the growing contribution of remotely generated internal fluctuations (interpretation supported by the kinetic energy budgets of section 5). Following this interpretation, approximately $80 \%$ of the baroclinic energy in the simulation on $\mathrm{g} 4$ can be accounted for by remote generation. For small domains ( $\mathrm{g} 1$ and g2), the control region barotropic energy is larger than the baroclinic one and increases by only a factor of 2.1 between $\mathrm{g} 1$ and $\mathrm{g} 4$. The barotropic contribution to the total energy for g3 and g4 is less than that of the baroclinic energy. The increase of barotropic kinetic energy is interesting in light of the negligible domain size sensitivity of the barotropic simulation energy level (gray line in Fig. 5). It is the signature of the adjustment of the barotropic 


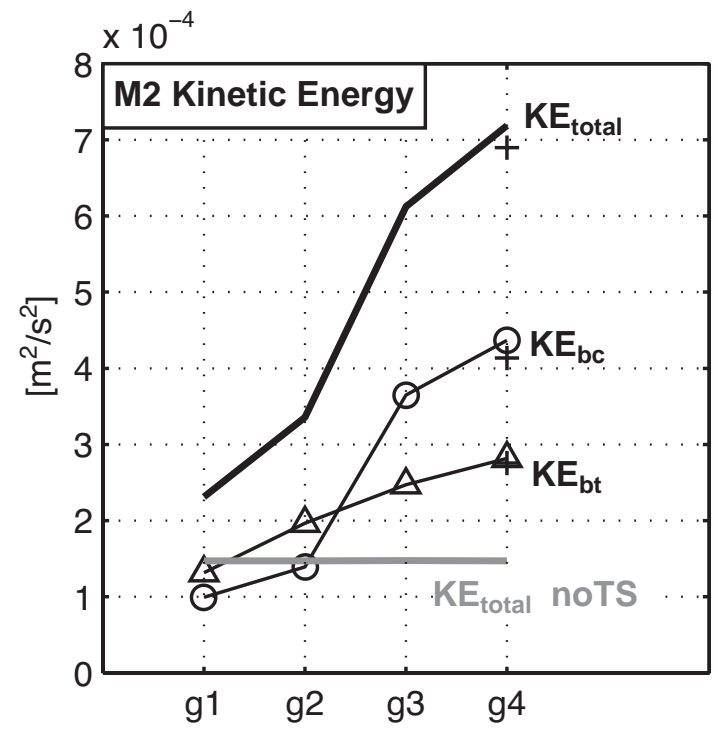

Figure 5: Control region total (full), baroclinic (circles) and barotropic (triangles) kinetic energies as a function of domain size and averaged over day 15. Black crosses $(+)$ represent the energy of the nested experiment (see section 4.3). The gray line shows the total kinetic energy of barotropic experiments (noTS).

dynamics to energy exchanges with the internal tide (confirmed by energy budgets in section 5.2). The total and baroclinic energy differences between g3 and g4 are less than the energy difference between smaller domains which may be an indication that the energy eventually plateaus. Computational resources did not allow consideration of larger domains at this resolution.

\subsection{Nested experiment}

The feasibility of a nested approach is tested with a simulation performed in domain g1 forced by boundary conditions obtained from the tidal solution in g4. Harmonic fits to sea level, three-dimensional currents and tracers over the last five days of the g4 simulation are used to prescribe the tidally fluctuating boundary conditions. Sea level is clamped at the boundary while the horizontal flow and tracers are relaxed at the boundaries as well as within adjacent nudging layers toward tidally fluctuating values consistent with g4 harmonic fits. Nudging layers are $5 \mathrm{~km}$ wide and the relaxation time scale is $1000 \mathrm{~s}$. The energy levels averaged inside the control region of the nested experiment are comparable to that of the simulation in domain g4 (Fig. 5). The total energy is weaker by approximately $4 \%$ and reflects a decrease in 
baroclinic energy. The control region volume-averaged squared difference between the flow from the nested simulation and that in domain $\mathrm{g} 4$ is about $3.5 \%$. A similar point-wise comparison for the barotropic flow indicates it is passed particularly well, to within $1 \%$ of the control region barotropic energy. The nested experiment reproduces tidal fluctuations of at least the first four baroclinic modes equally well. Projections of the flow of the nested model onto these modes are comparable to the projections of the flow in domain g4 within 3 to $5 \%$ of the spatially averaged energy represented by each mode. This experiment therefore confirms the feasibility of a nested approach provided appropriate boundary conditions are known.

\section{M2 Kinetic Energy budget and fluxes}

\subsection{Barotropic and baroclinic energy equations}

Budgets of the control region kinetic energy are used next to explain the kinetic energy trends described in section 4. We distinguish between barotropic and baroclinic energy budgets. Detailed derivations of the energy budget for barotropic and baroclinic flows are found in Zaron and Egbert (2006), Carter et al. (2008), and, Kelly et al. (2010). We start here with the 3D linear depth-integrated kinetic energy budget (Zaron and Egbert, 2006):

$$
\begin{aligned}
\partial_{t} \int_{-h}^{0} \rho_{0} \frac{\mathbf{u}^{2}}{2} d z+\nabla & \cdot \int_{-h}^{0}(p \mathbf{u}) d z=-p_{s} w(z=0) \\
& -g \int_{-h}^{0} \rho w d z-\rho_{0} \int_{-h}^{0} \epsilon_{K} d z-\mathbf{u}(z=-h) \cdot \tau_{b},
\end{aligned}
$$

where $\mathbf{u}=(u, v), \eta$ is the sea level, $p_{s}$ is the pressure at $z=0, \rho$ is the density minus its time average (which has a vertical structure), and, $p$ is the hydrostatic pressure:

$$
p=g \rho_{s} \eta+\int_{z}^{0} \rho g d z .
$$

From left to right, the terms involved in (3) are the time rate of change of kinetic energy, the divergence of the pressure work, the surface and interior conversions between kinetic energy and potential energy, and, the interior ( $\epsilon_{K}$ is the local rate of viscous dissipation) and bottom stress $\left(\tau_{b}\right)$ dissipation of kinetic energy. The time rate of change of kinetic energy and the surface 
conversion between kinetic and potential energy $\left(p_{s} w(z=0)=\rho_{s} g \partial_{t} \eta^{2} / 2\right.$, where $\rho_{s}$ is the density at the surface) average in time to zero.

The barotropic kinetic energy budget is obtained after multiplication of the depth-integrated momentum equations by the depth-averaged flow:

$$
\rho_{0} h \partial_{t}\left(\bar{u}^{2}+\bar{v}^{2}\right) / 2+\nabla \cdot(h \overline{\mathbf{u}} \bar{p})=-p_{s} \bar{w}(z=0)-g \int_{-h}^{0} \rho \bar{w} d z,
$$

where $\bar{w}$ is the vertical velocity associated with the depth-averaged flow:

$$
\bar{w}=(1+z / h) w(z=0)+\frac{z}{h} \overline{\mathbf{u}} \cdot \nabla h .
$$

Dissipative effects have been ignored here for simplicity. The barotropic energy loss due to bottom friction and horizontal dissipation was approximately estimated from an harmonic fit on depth-averaged current which lead to values of the order of $0.15 \mathrm{MW}$ ( $5 \%$ of the baroclinic energy dissipation for g4, Fig 6). The conversion of barotropic kinetic energy into available potential energy (labeled $\bar{C}$ ) is related to a classical definition of baroclinic conversion (Kelly et al., 2010):

$$
-g \int_{-h}^{0} \rho \bar{w} d z=p^{\prime}(z=0) \partial_{t} \eta+p^{\prime}(z=-h) \overline{\mathbf{u}} \cdot \nabla h .
$$

Subtracting (5) from (3) leads to the baroclinic kinetic energy budget:

$$
\begin{aligned}
& \partial_{t} \int_{-h}^{0} \rho_{0} \frac{\mathbf{u}^{\prime 2}}{2} d z+\nabla \cdot \int_{-h}^{0}\left(p^{\prime} \mathbf{u}^{\prime}\right) d z= \\
& -g \int_{-h}^{0} \rho w^{\prime} d z-\rho_{0} \int_{-h}^{0} \epsilon_{K} d z-\mathbf{u}(z=-h) \cdot \tau_{b} .
\end{aligned}
$$

In the preceding budget we have assumed that the total energy dissipation is due to baroclinic fluctuations, which is approximately true based on the amplitude of the barotropic energy dissipation. The conversion of available potential energy into baroclinic energy (labeled $C^{\prime}$ ) represents the local production of baroclinic kinetic energy and nearly equals $\bar{C}$. Both $\bar{C}$ and $C^{\prime}$ can therefore be taken as measures of "baroclinic conversion" (see Zaron and Egbert (2006) for a review of the various definitions). The difference between $\bar{C}$ and $C^{\prime}$ is due to mixing. Mixing indeed destroys available potential energy (Zaron and Egbert, 2006) and the amount of energy extracted from the 

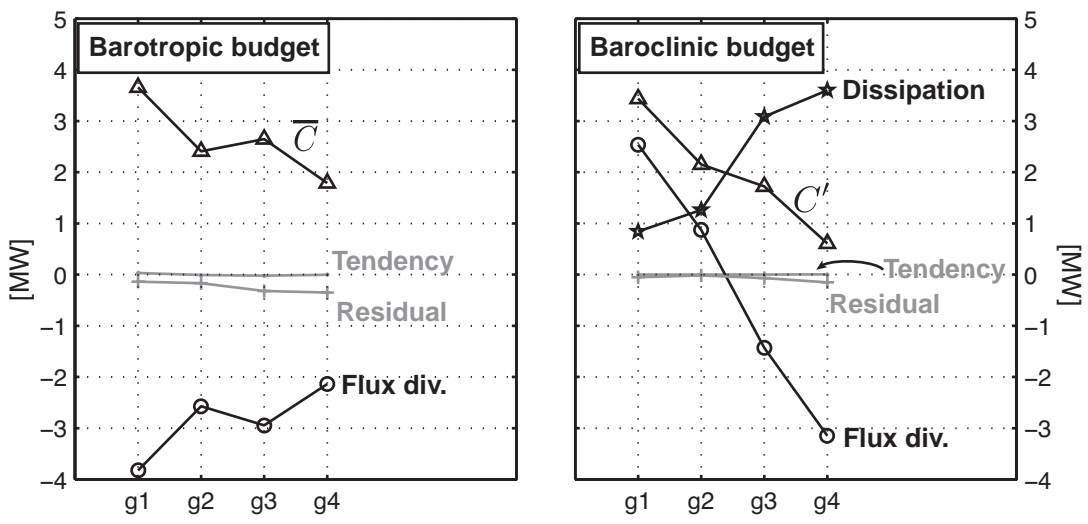

Figure 6: Barotropic (left) and baroclinic (right) kinetic energy budget for the control region. These budgets are averaged over the last 10 tidal cycles. Units are in megawatts.

barotropic field (measured by $\bar{C}$ ) is as a result slightly larger than the actual production of baroclinic kinetic energy (measured by $C^{\prime}$ ), as seen in Fig. 6 .

To summarize, the kinetic energy budgets can be written in the following form:

$$
\text { Tendency }+ \text { Flux div. }= \begin{cases}-\bar{C} & \text { (barotropic budget) } \\ C^{\prime}-\text { Dissipation } & \text { (baroclinic budget) }\end{cases}
$$

\subsection{Kinetic energy budget}

For the small domains (g1 and g2), there is a net outward flux of baroclinic energy (Fig. 6, right) from the control domain. The conversion $C^{\prime}$ is positive indicating a barotropic to baroclinic transfer of energy. The leading order balance is between this local conversion and its export at the boundaries of the control region, dissipation being of smaller magnitude. For larger domains (g3 and g4), the flux of energy reverses and there is a net inflow of baroclinic energy. The conversion term is still a transfer from barotropic to baroclinic energy but its magnitude has decreased. Such a trend would tend to produce smaller baroclinic and larger barotropic kinetic energy inside the control region for larger domains. While the local changes in conversion could therefore explain the observed increase of barotropic energy with domain size, they cannot explain that of baroclinic kinetic energy. In experiments g1 through g4, dissipation is proportional to baroclinic kinetic energy to within $10 \%$, and the proportionality constant $(\sim 0.9$ day $)$ may be interpreted as a damping time scale. For the two larger domains, the baroclinic 

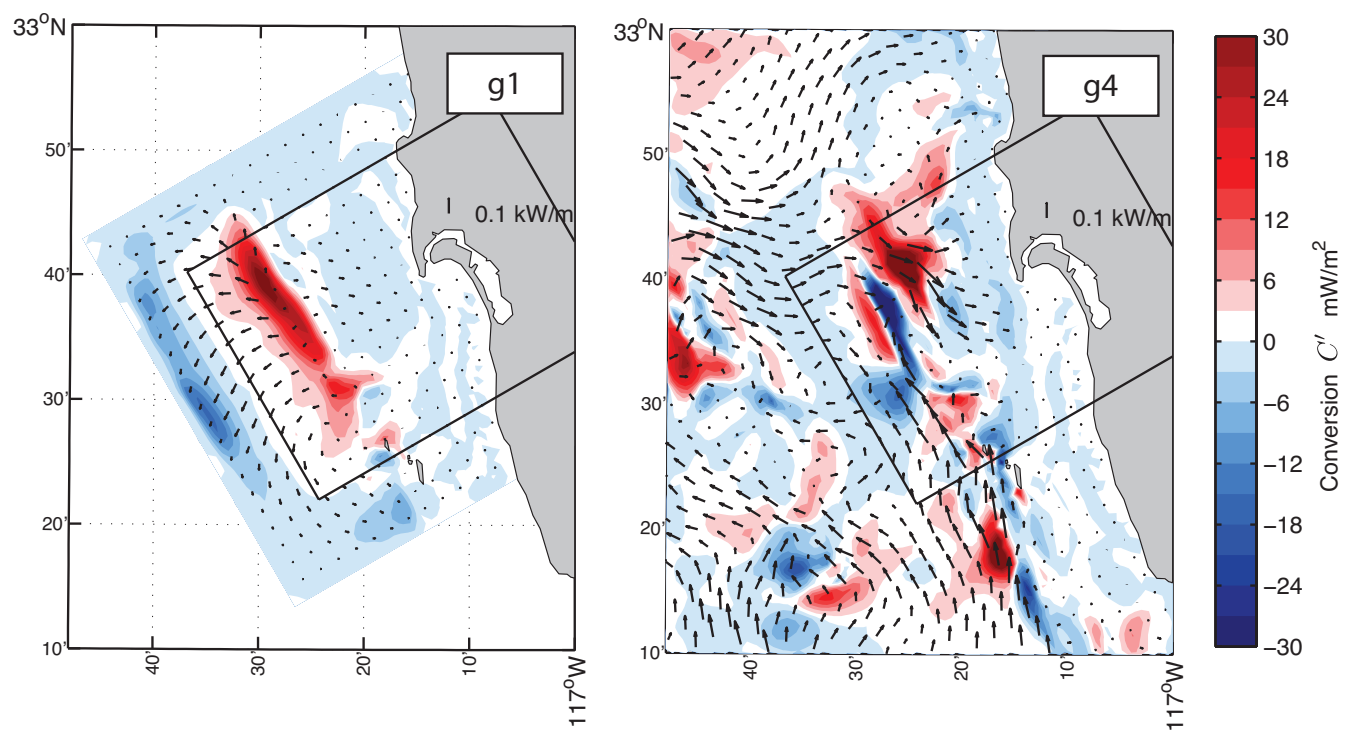

Figure 7: Baroclinic energy fluxes for domain g1 (left) and g4 (right). Color shows the conversion rate $C^{\prime}$. Variables are averaged over the last 10 tidal cycles.

kinetic energy budget has changed: the leading order balance is between the net flux of energy into the control region and dissipation. These budgets support the interpretation that the contribution of remotely generated internal fluctuations is growing with domain size and increases the control region baroclinic energy.

The control volume barotropic kinetic energy budget shows a balance between the flux divergence and conversion $\bar{C}$ (Fig. 6, left). The magnitude of both terms is decreasing for larger domains and confirms that, within the control volume, the barotropic dynamics indeed adjusts to the production of baroclinic energy.

\subsection{Local energy fluxes and conversion rate.}

Maps of the baroclinic conversion rate $\left(C^{\prime}\right)$ and of the depth-integrated fluxes of baroclinic energy provide further details of the domain size sensitivity in the neighborhood of the control region (Fig. 7). For domain g1, there is a well-defined area of baroclinic energy production located over the shelf break on the western part of the control region. Energy radiates from there and exits the control region through its western boundary. For domain $\mathrm{g} 4$, the distribution of baroclinic conversion is strongly modified. There is 
now an area of negative conversion over the shelf break which explains the decrease of the conversion rate with domain size observed in Fig. 6. Positive baroclinic conversion occurs at the foot of the shelf break as well as over a trough located at the north of the control region (see Fig. 1 for the bathymetry). Energy fluxes show that energy flows into the control region from several directions. Spatial structures of the conversion rate and of the fluxes are more complex than for domain g1.

As explained in section 5.1, $C^{\prime}$ and $\bar{C}$ are nearly equal and their sensitivity to domain size are therefore found to be comparable (Fig. 6). Changes to $\bar{C}$ are attributed to either changes in tidal fluctuations of density, changes of barotropic vertical velocity, or, the combined changes of density and barotropic velocity. This can be seen for example from (7) and a decomposition of the local, instantaneous changes in vertical mass flux from the simulation in domain $\mathrm{g} 1$ to the simulation in domain $\mathrm{g} 4$ :

$$
\rho_{4} \bar{w}_{4}-\rho_{1} \bar{w}_{1}=\bar{w}_{1} \delta \rho+\rho_{1} \delta \bar{w}+\delta \rho \delta \bar{w}
$$

where $\delta \rho=\rho_{4}-\rho_{1}$ and $\delta \bar{w}=\bar{w}_{4}-\bar{w}_{1}$. From domain g1 to domain g4, $\bar{C}$ decreases by about $1.9 \mathrm{MW}$ (Fig. 6, left). Changes in density are such that they would decrease the conversion by 6.0 MW, thereby limiting the local production of baroclinic energy. This is counterbalanced by a $4.4 \mathrm{MW}$ increase due to changes of the barotropic vertical velocity field. Combined changes in density and vertical velocity only account for a 0.3 MW decrease. Other studies have investigated the role of remotely generated internal tide on conversion (Kelly and Nash, 2010; Kelly et al., 2012). The present situation differs from these studies in that there is an adjustment of the barotropic dynamics which contribute to the observed changes in conversion.

\subsection{Remote conversion rate.}

Changes in barotropic dynamics from one simulation to another can affect the control volume baroclinic kinetic energy in several ways. First by modifying the local conversion of barotropic to baroclinic energy. In section 5.3, we have seen that changes of the tidal barotropic vertical velocity contributes to the total change in conversion. However, the change of conversion with increasing domain size would tend to decrease the control volume baroclinic kinetic energy, which is not what is observed (section 4.2).

Changes in the barotropic dynamics could also affect the remote (i.e. outside the control region) generation of baroclinic energy. To assess this 


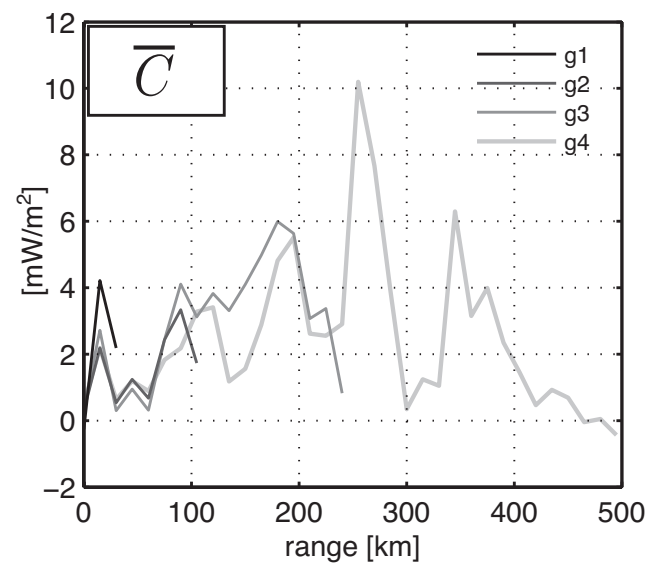

Figure 8: Conversion rate $\bar{C}$ averaged in $15 \mathrm{~km}$ wide rings around the center of the control region and as a function of distance from this center. Gray levels corresponds to different numerical domain sizes.

issue, $\bar{C}$ is averaged in $15 \mathrm{~km}$ wide rings around the center of the control volume and shown as a function of range in Fig. 8. The conversion fluctuates between $1 \mathrm{~mW} \mathrm{~m}^{-2}$ and $10 \mathrm{~mW} \mathrm{~m}^{-2}$ (spatial means are 3.0 and $3.2 \mathrm{~mW} \mathrm{~m}^{-2}$ for $\mathrm{g} 4$ and g3 respectively). The peak of conversion around $250 \mathrm{~km}$ range is the signature of the strong internal tide generation that takes place inside the Santa Cruz Basin (Buijsman et al., 2012). Conversion rates with domains g1, g2 and g3 are at most within a factor of two from the conversion estimate on grid g4. Between 100 and $200 \mathrm{~km}$ range, the magnitude of the conversion with g4 is lower than that with g3. From the simulation in domain g3 to that in domain $\mathrm{g} 4, \bar{C}$ averaged over domain $\mathrm{g} 3$ decreases by $0.20 \mathrm{~mW} \mathrm{~m}^{-2}$. This change is the sum of contributions due to changes of fluctuations of density $\left(-0.17 \mathrm{~mW} \mathrm{~m}^{-2}\right)$, changes of barotropic vertical velocities $\left(-0.22 \mathrm{~mW} \mathrm{~m}^{-2}\right)$ and to their combined changes $\left(0.19 \mathrm{~mW} \mathrm{~m}^{-2}\right)$. We conclude that, while there are modest changes of the conversion rate from one grid to another, it is unlikely that the increase in baroclinic kinetic inside the control volume is due to an intensification of the conversion. It is more likely that this increase results from the growing number of internal tide generation sites included within larger domains. 


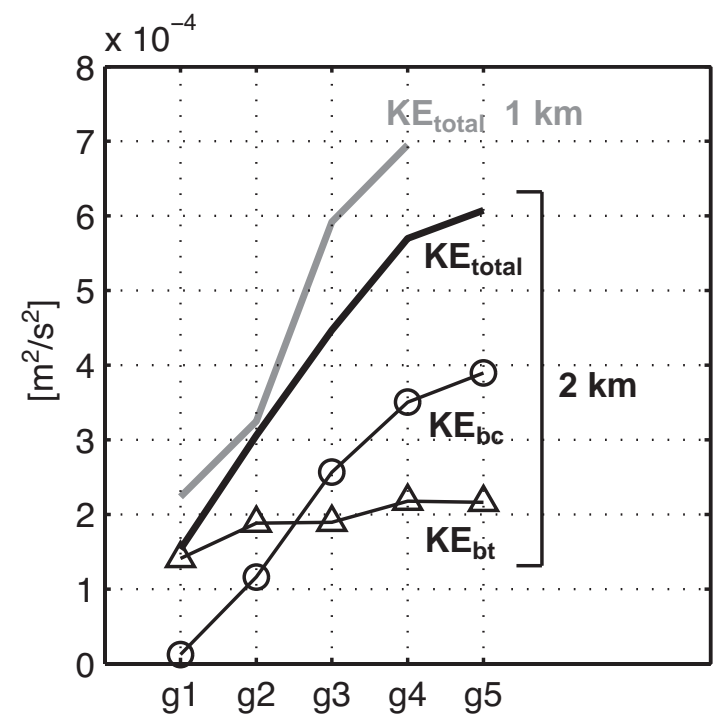

Figure 9: Control region kinetic energy as a function of domain size with a $2 \mathrm{~km}$ grid spacing in black and with $1 \mathrm{~km}$ grid spacing in gray. Total kinetic energy (full lines) and its baroclinic (circles) and barotropic (triangles) contributions are shown. Energy is averaged over the last day.

\section{On the choice of grid cell size and viscosity.}

\subsection{Effect of a coarser grid on the M2 kinetic energy}

In light of the continuing increase of kinetic energy, the size of the numerical domain should ideally be increased until no further increase was seen. The grid spacing was increased to $2 \mathrm{~km}$, to allow simulations with domains up to $1024 \times 1024 \mathrm{~km}$, referred to as g5. The vertical spacing was doubled in order to keep a consistent grid aspect ratio (following Zaron and Egbert (2006)) and viscosities were increased so as to keep the ratios $K_{v} / d z^{2}$ and $K_{h} / d x^{2}$ constant. The control region is at the same geographical location as for the $1 \mathrm{~km}$ grid.

For numerical domains of comparable area, the control region kinetic energy is lower by as much as $20 \%$ with $2 \mathrm{~km}$ grid spacing than with $1 \mathrm{~km}$ spacing (Fig. 9). This is consistent with past research which has shown that the use of coarser grids reduces the production of internal tide (Zaron and Egbert, 2006; Carter et al., 2008; Zilberman et al., 2009). Indeed, the spatially averaged conversion $\bar{C}$ with a $2 \mathrm{~km}$ grid spacing is $38 \%$ that with a $1 \mathrm{~km}$ grid spacing. On the other end of the internal tide life cycle, the 
use of a coarser grid may also affect the internal tide propagation and decay, which is believed to occur via scattering off bathymetry into small scale internal waves (Müller and Bühler, 2009). Arguably, the use of a subgrid scale parametrization appropriate to grid spacings comparable to the present one, i.e. when internal wave breaking is not resolved, should dissipate resolved scales equally well when grid spacing is varied. In the present case however, the prescribed increase of viscosities with $2 \mathrm{~km}$ grid spacing more strongly damps internal tide fluctuations of any wavelength. This ultimately limits the contributions from remote baroclinic generation sites and could be another explanation for the decreased energy with a $2 \mathrm{~km}$ grid spacing.

Despite the overall decrease of energy, the trends of total, barotropic and baroclinic kinetic energies are comparable to that with $1 \mathrm{~km}$ grid spacing for domains g1 to g4. The difference of total kinetic energy between g1 and g4 is $4.710^{-4} \mathrm{~m}^{2} \mathrm{~s}^{-2}$ for $1 \mathrm{~km}$ grid spacing against $4.2510^{-4} \mathrm{~m}^{2} \mathrm{~s}^{-2}$ for $2 \mathrm{~km}$ grid spacing. This slightly slower rate of increase of kinetic energy likely results from the reduced internal tide production with coarser grids and the stronger damping of internal fluctuations. Between $\mathrm{g} 4$ and g5, the increase of kinetic energy is remarkably slower than for the smaller domains. Numerical simulations on larger domains would be required to confirm a plateau is within reach.

\subsection{Effect of increased viscosities on the M2 kinetic energy}

We conduct next a sensitivity experiment on the viscous energy loss by doubling horizontal and vertical viscosities. The expectation is that it will hasten the decay of remotely generated fluctuations and lead to lower levels of baroclinic energy inside the control region. Reports on the effect of turbulence parametrization for a regional tidal simulations are sparse. Niwa and Hibiya (2004) found that increasing viscosities and diffusivities did not alter the rate of conversion $\bar{C}$ but increased kinetic energy dissipation in a simulation of the East China Sea. Note that this would not happen in an enclosed area where the volume averaged conversion rate has to balance dissipation. Kang and Fringer (2012) find that conversion and the inferred energy dissipation (i.e. the residual of the energy budget) is insensitive to prescribed viscosities. The experiments of Kang and Fringer (2012) however differ from the present ones as the bulk of their energy dissipation is numerical.

The total amount of energy inside the control region is less ( $15 \%$ for the largest domain g4) than that with the base choice of viscosities (Fig. 10). This decrease is the reflection of an equal drop of baroclinic energy. Along 


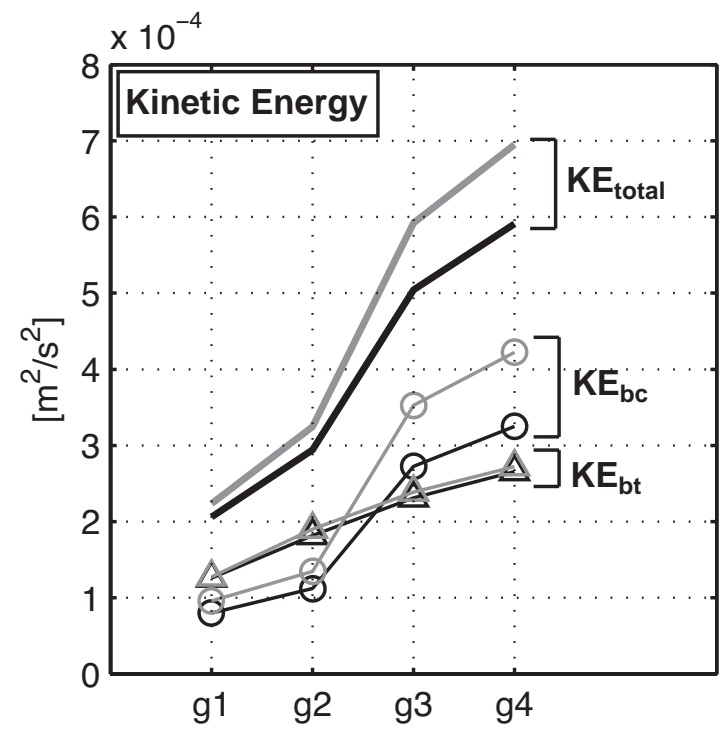

Figure 10: Control region kinetic energy as a function of domain size for the base choice of viscosities (in grey) and for doubled viscosities (in black). Total kinetic energy (full lines) and its baroclinic (circles) and barotropic (triangles) contributions are shown. The energy is averaged over the last day.

with the decreased baroclinic energy flux into the control region (not shown), these observations confirm the initial expectation that the decay of remotely generated internal fluctuations has been hastened by the larger viscosities. The barotropic energy, on the other hand, is not as affected by the increased viscosities. This is consistent with Niwa and Hibiya (2004), Kang and Fringer (2012), and the constancy of barotropic to baroclinic conversion when viscosities and/or diffusivities are increased.

Ideally, one would like to use viscosities and diffusivities appropriate to our choice of grid spacing (i.e. too coarse to resolve wave breaking), the regimes of internal wave activity at hand, and potentially the unresolved bathymetric distribution. Bottom boundary layers have potential importance on internal tide generation (Kurapov et al., 2010) and should also be treated more realistically. An alternative would be to greatly reduce grid sizes and resolve the breaking of internal waves but this would require an unrealistic amount of computational power. We can therefore only conclude here that, even with our base case viscosities, it is very likely that we are overestimating the damping of remotely generated internal fluctuations and that the growth 


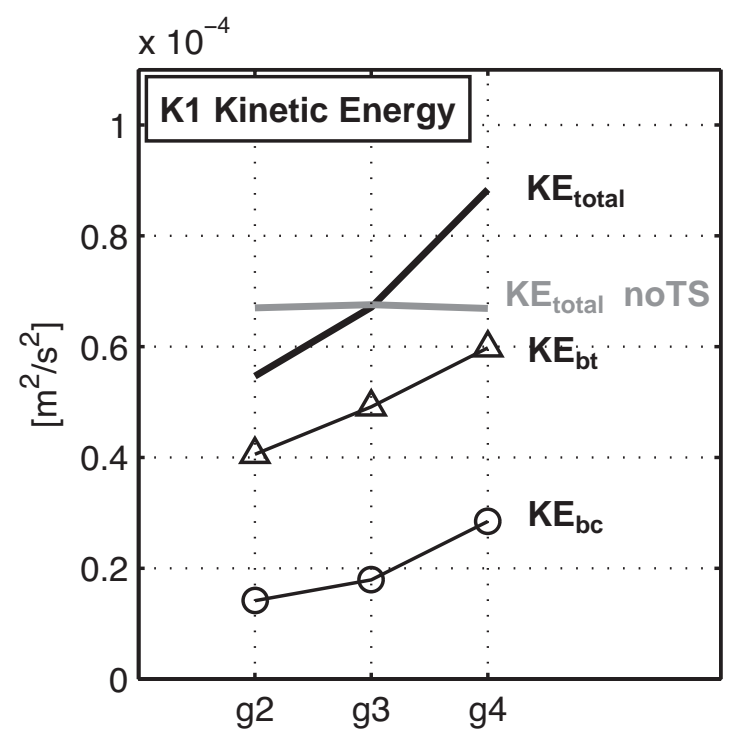

Figure 11: K1 tide. Control region kinetic energy as a function of domain size. The total kinetic energy (full lines) and its baroclinic (circles) and barotropic (triangles) contributions are shown. The kinetic energy is averaged over the last 5 days.

of the control region kinetic energy only stops for domains larger than those considered here.

\section{K1 tidal kinetic energy.}

Diurnal tidal frequencies such as K1 are subinertial in the Southern California Bight. The nature of the tidal response is different at subinertial frequencies as internal gravity waves are trapped against topographic features and cannot propagate freely (Brink, 1991; Dale and Sherwin, 1996). This trapping can lead to amplified responses close to isolated topographic features and enhanced local mixing (Kunze and Toole, 1997; Tanaka et al., 2010). The limited propagation of diurnal internal tide should be an advantage for the tidal modeling of these constituents. We check this now by forcing the model with the K1 constituent. K1 sea level fluctuations are about $34 \mathrm{~cm}$ in the control region (against $50 \mathrm{~cm}$ for $\mathrm{M} 2$ ). Despite their evanescent nature, internal fluctuations reach some distance off their generation sites. As a result, a spurious interaction occurs in the baroclinic simulation in domain g1 between the western boundary nudging layer and internal fluctuations generated along the shelf break near the control domain 
western boundary. Adjustments of the nudging layer width or the relaxation time scale did not get rid of this issue and we therefore do not show results in domain g1 for K1 simulations. Finally, simulations are 30 days long (compared to 15 days for M2) in order to accommodate for a slower spin up of K1 fluctuations. Kinetic energy levels are estimated over the last 5 days of the simulations.

For barotropic runs, the control region kinetic energy (gray in Fig. 11) varies very weakly with domain size, to within less than $1 \%$ of its average level. For baroclinic runs, the kinetic energy is predominantly barotropic inside the control region, with a ratio of barotropic to total energy between $67 \%$ and $73 \%$ (Fig. 11). This is a marked difference with the M2 case, where the increase of baroclinic kinetic energy with increasing numerical domain size produced a large variation of the ratio of baroclinic to barotropic energy (see section 4.2). There is an overall increase of total kinetic energy (60\% of the g2 level) explained by the simultaneous growths of barotropic and baroclinic energies. This increase is weaker than that for the M2 tide, as the M2 total kinetic energy nearly doubles inside the control region from g2 to g4 simulations (Fig. 5).

Barotropic kinetic energy budgets (not shown) indicate that there is some adjustment of the barotropic dynamics. The barotropic energy fluxes are approximately parallel to the control domain western boundary but slightly veer into the boundary for larger domains. There is consequently a growth of the convergence of barotropic energy fluxes inside the control domain which is counterbalanced by an increase of barotropic to baroclinic conversion. As in the case of the M2 tide, this confirms that the barotropic tidal fluctuations adjust locally to the possible conversion of barotropic to baroclinic energy. The increase in the conversion $C^{\prime}$ is about $0.18 \mathrm{MW}$ from g2 to g4 and could explain the growth of baroclinic kinetic energy with domain size. The flux of incoming baroclinic energy through the southern boundary is however also increasing with domain size, albeit in a smaller extent (0.07 MW). This increased flux is consistent with the signature of coastal-trapped waves (Hutnance, 1992) which can accumulate an increasing amount of energy along the coastline for larger domains.

\section{Conclusion}

This study computed the sensitivity to numerical domain size of the tidal response inside a control region along the Southern California Bight coastline. 
Unlike sea level, which varies weakly, the kinetic energy was found to increase with domain size. This coincided with increases of the incoming flux of baroclinic energy through the control region boundaries, which suggests that the growing contribution from remotely generated internal fluctuations is responsible. Some variations of the barotropic to baroclinic conversion were observed with domain size and were explained by both changes in fluctuations of the density and barotropic vertical velocity. Neither local (i.e. within the control region) nor remote (i.e. outside the control region) changes of conversion seemed consistent with the observed increase of baroclinic kinetic energy. We also showed with a nested experiment that proper knowledge of the 3D tidal fluctuations along the smallest domain boundaries is sufficient to reproduce the elevated energy levels obtained with the runs on the largest domain.

Eventually we would expect the kinetic energy increase to stop when the most distant fluctuations are scattered and dissipated away before reaching the control region. While this follows intuition, it is unclear a priori for what domain size this actually would occur. To answer that question would require estimating both the intensity of the generation as well as the damping rate of the fluctuations.

To test the proposed interpretation, viscosities were doubled, resulting in lower baroclinic energy inside the control region, consistent with stronger damping of remotely generated fluctuations and a decrease of their contributions. Coarser grids allowed the use of larger domains and the increase of kinetic energy with domain size slowed somewhat for the $1024 \times 1024 \mathrm{~km}$ domain. The present study cannot conclude on the importance of internal wave sources located more than $1000 \mathrm{~km}$ away from the control region.

We expect the issue posed by contributions from remotely generated fluctuations to be generic even though our conclusions are specific to the particularities of the domain considered (mostly but not exclusively the surrounding bathymetry). Background flow was ignored in these experiments, but could affect the generation and propagation of the remotely generated baroclinic tide and modulate the present results. The perturbation introduced by background flows are, for example, believed to be responsible for local fluctuations of the observed internal tide (Chavanne et al., 2010; Kelly and Nash, 2010; Zilberman et al., 2011; Kelly et al., 2012).

Finally, the tidal response for a subinertial constituent (K1) was computed. Some sensitivity to domain size was found with, in particular, a growth of kinetic energy inside the control region. This sensitivity is how- 
ever reduced compared to the M2 case, which is as expected given the limited propagation of internal fluctuations at subinertial frequencies.

\section{Acknowledgments}

A. L. Ponte was funded by the Office of Naval Research (ONR) grant number N00014-10-1-0990. B. Cornuelle was funded by ONR N00014-08-10587. Thanks to Kraig Winters, Jen MacKinnon and Shaun Johnston for fruitful discussions about this work.

\section{References}

Alford, M. H., MacKinnon, J. A., Zhao, Z., Pinkel, R., Klymak, J. M., Peacock, T., 2007. Internal waves across the Pacific. Geophysical Research Letters 34 (L24601).

Alford, M. H., Zhao, Z., 2007. Global Patterns of Low-Mode Internal-Wave Propagation. Part II: Group Velocity. J. Phys. Oceanogr. 37, 1849-1858.

Amante, C., Eakins, B. W., 2009. ETOPO1 1 Arc-Minute Global Relief Model: Procedures, Data Sources and Analysis. Tech. Rep. Technical Memorandum NESDIS NGDC-24, 19pp, NOAA.

Antonov, J. I., Locarnini, R. A., Boyer, T. P., Mishonov, A. V., Garcia, H. E., 2006. World Ocean Atlas 2005, Volume 2: Salinity. NOAA Atlas NESDIS 62, U.S. Government Printing Office, Washington, D.C., 182 pp.

Bratkovich, A., 1985. Aspects of the Tidal Variability Observed on the Southern California Continental Shelf. J. Phys. Oceanogr. 15, 225-239.

Brink, K. H., 1991. Coastal-trapped waves and wind-driven currents over the continental shelf. Annual Review of Fluid Mechanics 23, 389-412.

Bühler, O., Holmes-Cerfon, M., 2011. Decay of an internal tide due to random topography in the ocean. J. Fluid Mech. 678, 271-293.

Buijsman, M. C., Uchiyama, Y., McWilliams, J. C., Hill-Lindsay, C. R., 2012. Modeling Semidiurnal Internal Tide Variability in the Southern California Bight. J. Phys. Oceanogr. 42, 62-77. 
Carter, G. S., 2010. Barotropic and Baroclinic $M_{2}$ Tides in the Monterey Bay Region. J. Phys. Oceanogr. 40, 1766-1783.

Carter, G. S., Merrifield, M. A., Becker, J. M., Katsumata, K., Gregg, M. C., Luther, D. S., Levine, M. D., Boyd, T. J., Firing, Y. L., 2008. Energetics of $M_{2}$ Barotropic-to-Baroclinic Tidal Conversion at the Hawaiian Islands. J. Phys. Oceanogr. 38, 2205-2223.

Chavanne, C., Flament, P., Luther, D., Gurgel, K.-W., 2010. The surface expression of semi-diurnal internal tides near a strong source at hawaii. part ii: Interactions with mesoscale currents. J. Phys. Oceanogr. 40, 11801200 .

Dale, A. C., Sherwin, T. J., 1996. The Extension of Baroclinic CoastalTrapped Wave Theory to Superinertial Frequencies. J. Phys. Oceanogr. 26, 2305-2315.

Duda, T. F., Preisig, J. C., 1999. A Modeling Study of Acoustic Propagation Through Moving Shallow-Water Solitary Wave Packets. IEEE Journal of Oceanic Engineering 24 (1).

Dushaw, B., Cornuelle, B., Worcester, P., Howe, B., Luther, D., 1995. Barotropic and Baroclinic Tides in the Central North Pacific Ocean Determined from Long-Range Reciprocal Acoustic Transmissions. Journal of Physical Oceanography 25 (4), 631-648.

Egbert, G. D., Ray, R. D., 2001. Estimates of $\mathrm{M}_{2}$ tidal energy dissipation from TOPEX/Poseidon altimeter data. J. Geophys. Res. 106 (C10).

Egbert, G. D., Ray, R. D., 2003. Semi-diurnal and diurnal tidal dissipation from TOPEX/Poseidon altimetry. Geophysical Research Letters 30 (17).

Garrett, C., Kunze, E., 2007. Internal Tide Generation in the Deep Ocean. Annual Review of Fluid Mechanics, 57-87.

Hall, R. A., Carter, G. S., 2011. Internal Tides in Monterey Submarine Canyon. J. Phys. Oceanogr. 41, 186-204.

Hazewinkel, J., Winters, K. B., 2011. PSI of the Internal Tide on a $\beta$ Plane: Flux Divergence and Near-Inertial Wave Propagation. J. Phys. Oceanogr. 41, 1673-1682. 
Heathershaw, A. D., 1985. Some observations of internal wave current fluctuations at the shelf-edge and their implications for sediment transport. Cont. Shelf Res. 4 (4), 485-493.

Hoteit, I., Cornuelle, B., Kim, S. Y., Forget, G., Köhl, A., Terrill, E., 2009. Assessing 4D-VAR for dynamical mapping of coastal high-frequency radar in San Diego. Dynamics of Atmospheres and Oceans 48, 175-197.

Hutnance, J. M., 1992. Extensive slope currents and the ocean-shelf boundary. Prog. Oceanog. 29, 161-196.

Inall, M. E., Shapiro, G. I., Sherwin, T. J., 2001. Mass transport by nonlinear internal waves on the Malin Shelf. Cont. Shelf Res. 21, 1449-1472.

Kang, D., Fringer, O., 2012. Energetics of Barotropic and Baroclinic Tides in the Monterey Bay Area. J. Phys. Oceanogr. 42, 272-290.

Kelly, S. M., Nash, J. D., 2010. Internal-tide generation and destruction by shoaling internal tides. Geophysical Research Letters 37 (L23611).

Kelly, S. M., Nash, J. D., Kunze, E., 2010. Internal-tide energy over topography. J. Geophys. Res. 115.

Kelly, S. M., Nash, J. D., Martini, K. I., Alford, M. H., Kunze, E., 2012. The Cascade of Tidal Energy from Low to High Modes on a Continental Slope. J. Phys. Oceanogr. 42, 1217-1232.

Klymak, J. M., Moum, J. N., Nash, J. D., Kunze, E., Girton, J. B., Carter, G. S., Lee, C. M., Sanford, T. B., Gregg, M. C., 2006. An Estimate of Tidal Energy Lost to Turbulence at the Hawaiian Ridge. J. Phys. Oceanogr. 36, 1148-1164.

Kunze, E., Toole, J. M., 1997. Tidally Driven Vorticity, Diurnal Shear, and Turbulence atop Fieberling Seamount. J. Phys. Oceanogr. 27, 2663-2693.

Kurapov, A. L., Allen, J. S., Egbert, G. D., 2010. Combined Effects of WindDriven Upwelling and Internal Tide on the Continental Shelf. J. Phys. Oceanogr. 40, 737-756.

Kurapov, A. L., Egbert, G. D., Allen, J. S., Miller, R. N., Erofeeva, S. Y., Kosro, P. M., 2003. The $M_{2}$ Internal Tide off Oregon: Inferences from Data Assimilation. J. Phys. Oceanogr. 33, 1733-1757. 
Laurent, L. S., Garrett, C., 2002. The Role of Internal Tides in Mixing the Deep Ocean. J. Phys. Oceanogr. 32, 2882-2899.

Legg, S., Huijts, K. M. H., 2006. Preliminary simulations of internal waves and mixing generated by finite amplitude tidal flow over isolated topography. Deep Sea Research II 53, 140-156.

Legg, S., Klymak, J. M., 2008. Internal Hydraulic Jumps and Overturning Generated by Tidal Flow over a Tall Steep Ridge. J. Phys. Oceanogr. 38, 1949-1964.

Lerczak, J. A., Winant, C. D., Hendershott, M. C., 2003. Observations of the semidiurnal internal tide on the southern California slope and shelf. J. Geophys. Res. 108 (C3).

Locarnini, R. A., Mishonov, A. V., Antonov, J. I., Boyer, T. P., Garcia, H. E., 2006. World Ocean Atlas 2005, Volume 1: Temperature. NOAA Atlas NESDIS 61, U.S. Government Printing Office, Washington, D.C., $182 \mathrm{pp}$.

Lucas, A. J., Franks, P. J. S., Dupont, C. L., 2011. Horizontal internaltide fluxes support elevated phytoplankton productivity over the inner continental shelf. Limnol. Oceanogr. : Fluids \& Environment 1, 56-74.

Marshall, J., Adcroft, A., Hill, C., Perelman, L., Heisey, C., March 1997. A finite-volume, incompressible navier-stokes model for studies of the ocean on parallel computers. J. Geophys. Res. 102 (C3), 5753-5766.

Martini, K. I., Alford, M. H., Kunze, E., Kelly, S. M., Nash, J. D., 2011. Observations of Internal Tides on the Oregon Continental Slope. J. Phys. Oceanogr. 41, 1772-1794.

Müller, C. J., Bühler, O., 2009. Saturation of the Internal Tides and Induced Mixing in the Abyssal Ocean. J. Phys. Oceanogr. 39 (2077-2096).

Nash, J. D., Kunze, E., Toole, J. M., Schmitt, R. W., 2004. Internal Tide Reflection and Turbulent Mixing on the Continental Slope. J. Phys. Oceanogr. $34,1117-1134$.

Niwa, Y., Hibiya, T., 2004. Three-dimensional numerical simulation of $\mathrm{M}_{2}$ internal tides in the East China Sea. J. Geophys. Res. 109 (C04027). 
Noble, M., Jones, B., Hamilton, P., Xu, J., Robertson, G., Rosenfeld, L., Largier, J., 2009. Cross-shelf transport into nearshore waters due to shoaling internal tides in San Pedro Bay, CA. Cont. Shelf Res. 29, 1768-1785.

Pairaud, I. L., Auclair, F., Marsaleix, P., Lyard, F., Pichon, A., 2010. Dynamics of the semi-diurnal and quarter-diurnal internal tides in the Bay of Biscay. part 2: Baroclinic tides. Cont. Shelf Res. 30, 253-269.

Pineda, J., 1994. Internal tidal bores in the nearshore: Warm-water fronts, seaward gravity currents and the onshore transport of neustonic larvae. J. Mar. Res. 52, 427-458.

Ray, R., Mitchum, G., 1997. Surface manifestation of internal tides in the deep ocean: observations from altimetry and island gauges. Progress in Oceanography 40 (1-4), 135-162.

Roe, P. L., 1985. Some contributions to the modelling of discontinuous flows. In: Engquist, B. E., Osher, S., Somerville, R. C. J. (Eds.), LargeScale Computations in Fluid Mechanics. Vol. 22. Lect. Appl. Math., pp. 163193.

Rosenfeld, L., Shulman, I., Cook, M., Paduan, J., Shulman, L., 2009. Methodology for a regional model evaluation, with application to central California. Deep Sea Research II 56 (3-5), 199-218.

Sharples, J., Tweddle, J. F., Green, J. A. M., Palmer, M. R., Kim, Y.-N., Hickman, A. E., Holligan, P. M., Moore, C. M., Rippeth, T. P., Simpson, J. H., Krivtsov, V., 2007. Spring-neap modulation of internal tide mixing and vertical nitrate fluxes at a shelf edge in summer. Limnol. Oceanogr. 52 (5), 1735-1747.

Shroyer, E. L., Moum, J. N., Nash, J. D., 2010. Vertical heat flux and lateral mass transport in nonlinear internal waves. Geophysical Research Letters 37 (L08601).

Spargo, E. A., Westerink, J. J., Luettich, R. A. J., Mark, D. J., 2003. ENPAC 2003: A tidal constuent database for eastern north pacific ocean. Technical report erdc/chl tr-04-12, Coastal Inlets Research Program, U.S. Army Engineer Research and Development Center, Vicksburg, MS. 
Tanaka, Y., Hibiya, T., Niwa, Y., Iwamae, N., 2010. Numerical study of $\mathrm{K}_{1}$ internal tides in the Kuril straits. J. Geophys. Res. 115 (C09016).

Winant, C. D., Bratkovich, A. W., 1981. Temperature and Currents on the Southern California Shelf: A Description of the Variability. J. Phys. Oceanogr. 11, 71-86.

Zaron, E. D., Egbert, G. D., 2006. Verification studies for a z-coordinate primitive-equation model: Tidal conversion at a mid-ocean ridge. Ocean Modelling 14, 257-278.

Zhao, Z., Alford, M. H., 2009. New Altimetric Estimates of Mode-1 $M_{2}$ Internal Tides in the Central North Pacific Ocean. J. Phys. Oceanogr. 39, 1669-1684.

Zilberman, N. V., Becker, J. M., Merrifield, M. A., Carter, G. S., 2009. Model Estimates of $M_{2}$ Internal Tide Generation over Mid-Atlantic Ridge Topography. J. Phys. Oceanogr. 39, 2635-2651.

Zilberman, N. V., Merrifield, M. A., Carter, G. S., Luther, D. S., Levine, M. D., Boyd, T. J., 2011. Incoherent Nature of $M_{2}$ Tides at the Hawaiian Ridge. J. Phys. Oceanogr. 41, 2021-2036. 